\title{
Liver-specific NG37 overexpression leads to diet-dependent fatty liver disease accompanied by cardiac dysfunction
}

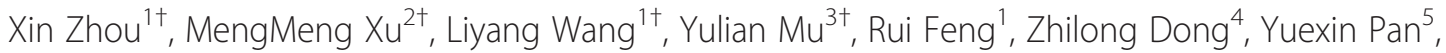
Xunzhang Chen ${ }^{6}$, Yongfeng Liu ${ }^{3,7}$, Shangen Zheng ${ }^{6}$, Donald D. Anthony ${ }^{5}$, Jianjie Ma ${ }^{8}$, Williams B. Isaacs ${ }^{9}$ and Xuehong $\mathrm{Xu}^{1 *}$

\begin{abstract}
Background: Environmental factors are well-known causes of diseases. However, aside from a handful of risk indicators, genes' encoding susceptibility to chronic illnesses and their associated environmental triggers are largely unknown. In this era of increasingly rich diets, such genetic predispositions would be immensely helpful from a public health perspective. The novel transgenic mouse model with liver-specific NG37 overexpression characterized in this article identifies the diet-dependent function of NG37 in the pathogenesis of fatty liver disease and cardiac arrhythmia.

Results: The liver-specific NG37 overexpression transgenic mouse model described here was generated using the Alb-SV40 polyA expression plasmid backbone. NG37 CDNA under control of the albumin promoter for liver-specific expression was fused with a $5^{\prime}$ terminal M2 FLAG sequence and a SV40 early region transcription terminator/polyadenylation site attached at the 3'-UTR. These NG37 transgenic mice developed normally and were physiologically normal on a standard diet. However, in comparison to non-transgenic (nTG) litter mates, these mice develop dramatic phenotypes within 12-18 days of starting a high-fat diet: (i) increased body weight $(28.5 \pm 12.3 \mathrm{~g})$, (ii) increased liver weight $(87.4 \pm 35.7 \mathrm{mg}$ ), (iii) increased heart weight (140 $\pm 38.4 \mathrm{mg}$ ), and (iv) cardiac arrhythmia. The enlarged livers of high-fat diet NG37 transgenic mice was histologically similar to human fatty liver disease and contained Maltese cross birefringent active depositions in hepatocytes that are indicative of fatty liver disease. We also confirmed via X-ray diffraction the steatotic vesicles in the diseased hepatocytes of our high-fat diet NG37 mice was composed of cholesteryl derivatives also found in human fatty liver disease. In addition to cardiac enlargement, NG37 transgenic mice on high-fat diet also exhibited highly irregular bradycardia not present in either high-fat diet nTG littermates or normal-diet transgenic litter mates.
\end{abstract}

Conclusions: The dramatic high-fat diet-dependent symptoms (increased body weight, cardiac enlargement, fatty liver, and cardiac arrhythmias) characterized in our liver-specific NG37 overexpression mouse model identifies NG37 as a gene encoding latent lipid metabolism pathology induced only in the presence of an environmental factor relevant to human health: high-fat diet.

Keywords: Cardiac dysfunction, Diet-dependent, Liver enlargement, NG37

\footnotetext{
* Correspondence: xhx0708@snnu.edu.cn

${ }^{\dagger}$ Equal contributors

${ }^{1}$ College of Life Sciences, Shaanxi Normal University, Xi'an, Shaanxi 710062,

China

Full list of author information is available at the end of the article
} 


\section{Background}

Diet is considered a causative factor in many human diseases such as cardiac disease, diabetes, and hypertension. We also know that genetics plays a significant role in determining susceptibility to these chronic illnesses $[1,2]$. However, two individuals with the same environmental risk factors (over-rich diet, sedentary lifestyle, etc.) may not both develop diabetes. While this difference in susceptibility to the disease is thought to be genetic, we do not yet know the specific genes responsible for this phenotypic difference. In this study, we report the ability of a high-fat diet to induce fatty liver disease and cardiac arrhythmia in mice with liver-specific overexpression of the NG37 gene.

NG37 encodes the NG37 protein, which is a member of the von Willebrand A (vWA) superfamily. Proteins from the vWA superfamily all contain a vWA domain and participate in a diverse range of cellular functions including transcription, DNA repair, and ribosomal/ membrane transport [3-6]. Him-4, the first vWA gene discovered via genetic analysis of Caenorhabditis elegans, encodes a vital extracellular matrix protein [3, 7, 8]. In mammals, this gene has two orthologs: fibulin-6/hemicentin-1 $(F 6 / H 1)$ and fibulin-8/hemicentin-2 (F8/H2), which encode fibulin-6/hemicentin-1 and fibulin-8/hemicentin2 , respectively. These two proteins share the vWA domain at their amino terminus with NG37. Recent studies have proven that these hemicentin proteins are required for successful mitosis during embryonic development [9-12]. However, despite the high homology of NG37 amino terminal region with these two previously characterized proteins, the actual function of NG37 is still unknown.

Our previous screening data have shown that NG37 is highly expressed in the liver at the RNA level, indicating that this gene could play a critical role in this vital organ. Thus, we chose to explore the function of NG37 in the liver by generating mice with NG37 overexpression driven by the liver-specific albumin promoter. Although these transgenic (TG) mice developed normally on a standard diet, when exposed to a high-fat diet, they rapidly developed pathologies. The most readily appreciated pathology was liver enlargement with hepatic lipid accumulation as identified by Maltese cross optical birefringence activity. This hepatosteatosis was confirmed by X-ray diffraction to be composed of the cholesterol accumulates also found in human fatty liver disease. In addition to fatty liver, these TG animals also developed severe cardiac arrhythmias likely as sequelae to disrupted lipid homeostasis. Our data suggest that overexpression of NG37 predisposes the mice to fatty hepatic enlargement and cardiac dysfunction when triggered by a fat-enriched diet. These findings identify a direct, previously unknown link between the intertwined roles of environmental factors and genetic predisposition in pathogenesis: the ability of high-fat diet to unmask a susceptibility to fatty liver disease encoded by the NG37 gene.

\section{Results and discussion}

Generation of the liver-specific Alb-NG37-overexpressing mouse model

As will be described in "Methods" section, the Alb-NG37 transgene construct (Fig. 1a) was injected into the pronucleus of zygotes collected from E0.5 day pregnant females. After brief culture in M16, zygotes were transferred into pseudopregnant females. After a full gestational term and natural birth, tail clippings from newborn pups were screened for the transgene by PCR (Fig. 1b).

To validate the liver-specific expression of NG37, liver samples were collected from Alb-NG37 transgenic mice and subsequently lysed for protein extraction. Immunoblotting with the M2-FLAG monoclonal antibody was used to confirm NG37 expression in the liver (Fig. 1c). Secondary confirmation with northern blot using a NG37 cDNA probe (M2-FLAG cDNA) confirmed that the minigene was only expressed in the liver and not in the heart of transgenic mice. We

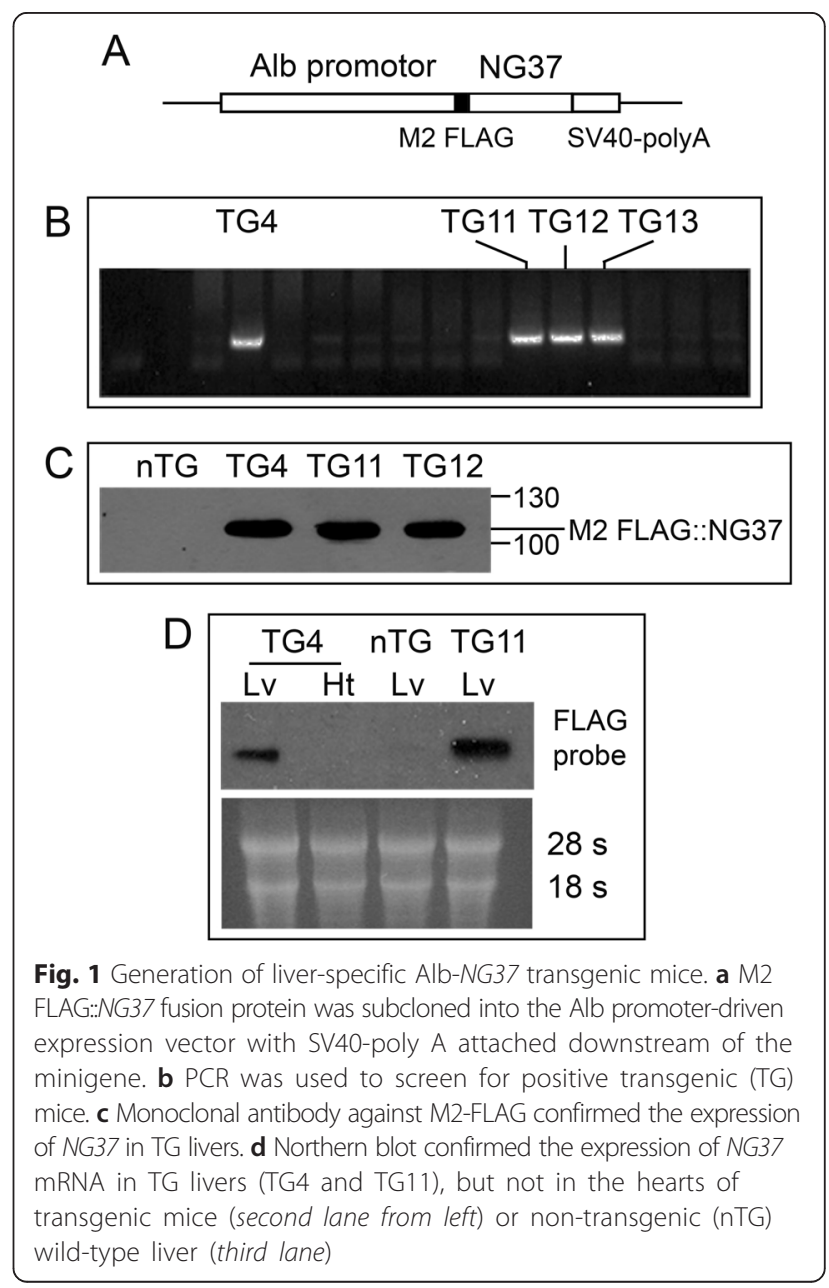


also confirmed that the minigene was not expressed in non-transgenic (nTG) littermate controls (Fig. 1d).

\section{Alb-NG37 TG mice developed normally and were indistinguishable from non-transgenic littermates} During phenotypic characterization of the Alb-NG37 TG mice, all mice were maintained on a normal diet and their growth curves, liver/body weight ratios, and heart/ body weight ratios were documented. Although TG mice had a slightly slower growth curve in comparison to their nTG littermate controls, mice achieved the same adult weight and the differences in growth curves were not statistically significant. This observation held true for both male (TG, $n=24$; nTG $n=13$ ) and female mice (TG, $n=24$; nTG, $n=16$ ) (Fig. 2a). There was also a slight and statistically insignificant increase in the liver/body weight ratio (male TG $5.11 \pm 1.81$; male nTG $4.92 \pm 1.71$; female TG $5.08 \pm 1.24$; female nTG $4.93 \pm 1.62$ ) and heart/ body weight ratio (male TG $4.90 \pm 1.70$; male nTG $4.89 \pm 1.32$; female TG $4.96 \pm 1.26$; female nTG $4.95 \pm 1.62$ ) of Alb-NG37 TG mice in comparison to nTG littermates at 4 months (Fig. 2b, c).

High-fat diet-triggered liver enlargement with liquid crystal lipid droplet accumulation in Alb-NG37 animals As previously discussed, Alb-NG37 mice fed a normal diet were phenotypically indistinguishable from nTG litter mates. However, a dramatic phenotype develops in NG37 transgenic animals when adult mice were switched to a high-fat (HiF) diet composed of the standard diet fried in a solution of $5 \%$ cholesterol homogenized in corn oil. Here, we note that while creating our HiF diet directly from regular chow may produce batch effects and slightly different conditions from the industry standard high-fat diets, the deep frying technique we used reflects the increase of deep fried foods in the human diet. Within 12-18 days of diet change, Alb-NG37 mice fed a HiF diet displayed a significant increase $(p<0.05)$ in body weight, liver/body weight ratio, and heart/body weight ratio. Body weights of HiF diet Alb-NG37 mice doubled in comparison to normal-diet Alb-NG37 and nTG littermates. More importantly, this dramatic phenotype remained just as true in a comparison between HiF diet Alb-NG37 mice and HiF diet nTG litter mates (Fig. 3a-c). Specifically, in comparison to normal-diet Alb-NG37 mice $(n=18)$, normal-diet nTG mice $(n=16)$, and HiF diet nTG mice $(n=16)$, HiF diet Alb-NG37 mice $(n=18)$ demonstrated a statistically significant $(p<0.05)$ gain of $28.5 \pm 12.3 \mathrm{~g}$ in body weight, $87.4 \pm 35.7 \mathrm{mg}$ in liver weight, and a doubling of heart weight at $140 \pm 38.4 \mathrm{mg}$ (Fig. 3d-f).

To characterize the enlarged livers, we assessed the histology and phase-transitional properties of liver sections from HiF diet Alb-NG37 mice and their HiF diet nTG littermates using a previously described protocol
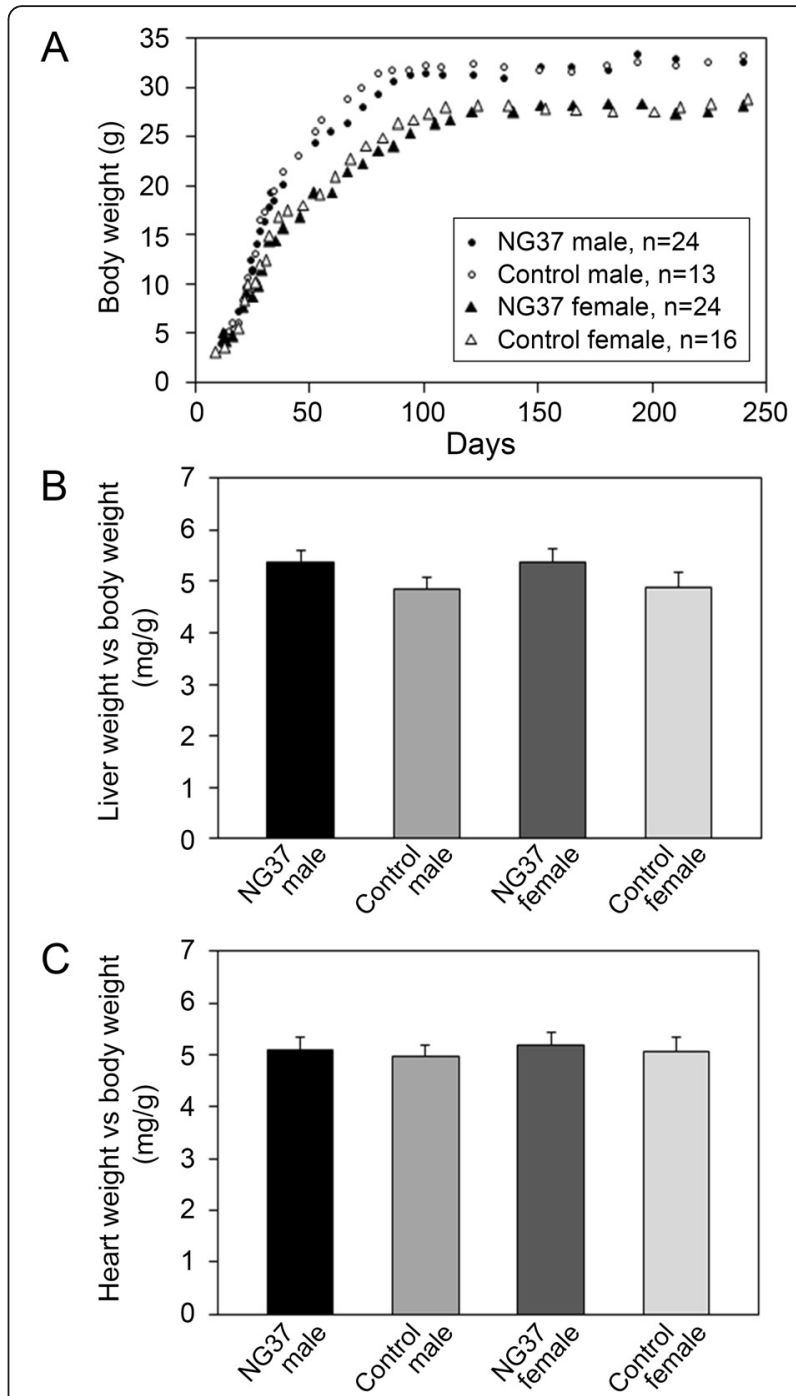

Fig. 2 Comparison of growth curve, liver/body weight ratio, and heart/body weight ratio of Alb-NG37 TG mice and nTG littermate controls. a Growth curve of the TG (male, $n=24$; female, $n=24$ ) and nTG (male, $n=13$; female, $n=16$ ) littermate control from birth to 8 months. $\mathbf{b}$ No biologically or statistically significant difference was seen between the liver weight/body weight ratios of male (TG $n=24$, nTG $n=13 ; p>0.05$ ) or female (TG $n=24$, nTG $n=16 ; p>0.05$ ) NG37 and $\mathrm{nTG}$ littermates. c No biologically or statistically significant difference was seen between the heart weight/body weight ratios of male (TG $n=24, n T G n=13 ; p>0.05$ ) and female (TG $n=24$, nTG $n=16 ; p>0.05)$ NG37 and nTG littermates. All analysis was performed on mice reared on a normal diet

for animal development [13-17]. While the livers of HiF diet Alb-NG37 mice retained normal liver structure on hematoxylin and eosin staining, the hepatocytes were enlarged and reticulated with large and small aggregates of steatosis (Fig. 4a) not seen in the livers of HiF diet nTG littermates (Fig. 4c), indicating that HiF diet AlbNG37 mice suffered from fatty liver disease. This was confirmed by the fact that HiF diet Alb-NG37 hepatocytes contained liquid crystal Maltese crosses (Fig. 4b) 

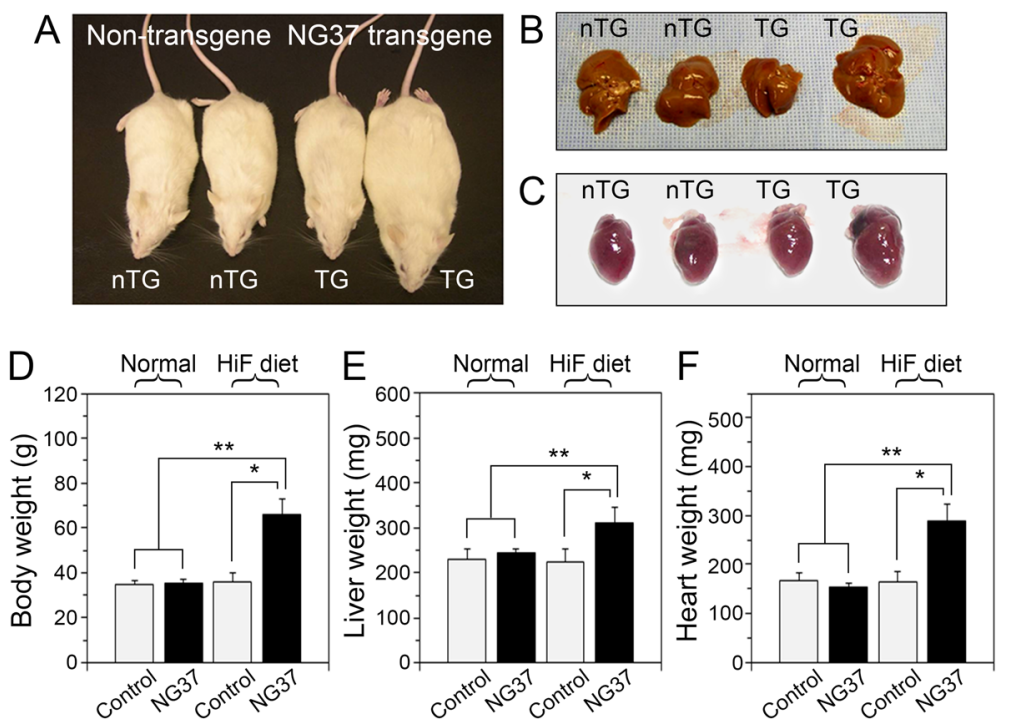

Fig. 3 HiF diet Alb-NG37 mice developed dramatic phenotypes in comparison to HiF diet nTG, normal-diet Alb-NG37, and normal-diet nTG littermates. a Gross differences in body size are observed in HiF diet Alb-NG37 mice (TG\#2) in comparison to normal-diet Alb-NG37 (TG\#1), normal-diet nTG (nTG\#1), and HiF diet nTG littermates (nTG\#2). Weight increase quantified in panel $\mathbf{d}$ notes that body weight of HiF diet TG mice $(n=18)$ significantly exceeded that of normal-diet TG and nTG mice $(n=18$ each, $p<0.01)$ and HiF diet nTG mice $(n=16, p<0.05)$. HiF diet Alb-NG37 mice also exhibited liver enlargement (b) and cardiac enlargement (c) quantified as organ weight relative to body weight (e, $\mathbf{f}$, respectively). e Liver weight of HiF diet TG mice $(n=18)$ significantly exceeded that of normal-diet TG and nTG mice $(n=18$ each, $p<0.01)$ and HiF diet nTG mice $(n=16, p<0.05)$. $\mathbf{f}$ Heart weight of HiF diet TG mice $(n=18)$ significantly exceeded that of normal-diet TG and nTG mice $(n=18$ each, $p<0.01)$ and HiF diet nTG mice (in e, $n=16, p<0.05$ ). ${ }^{* *} p<0.01 ;{ }^{*} p<0.05$
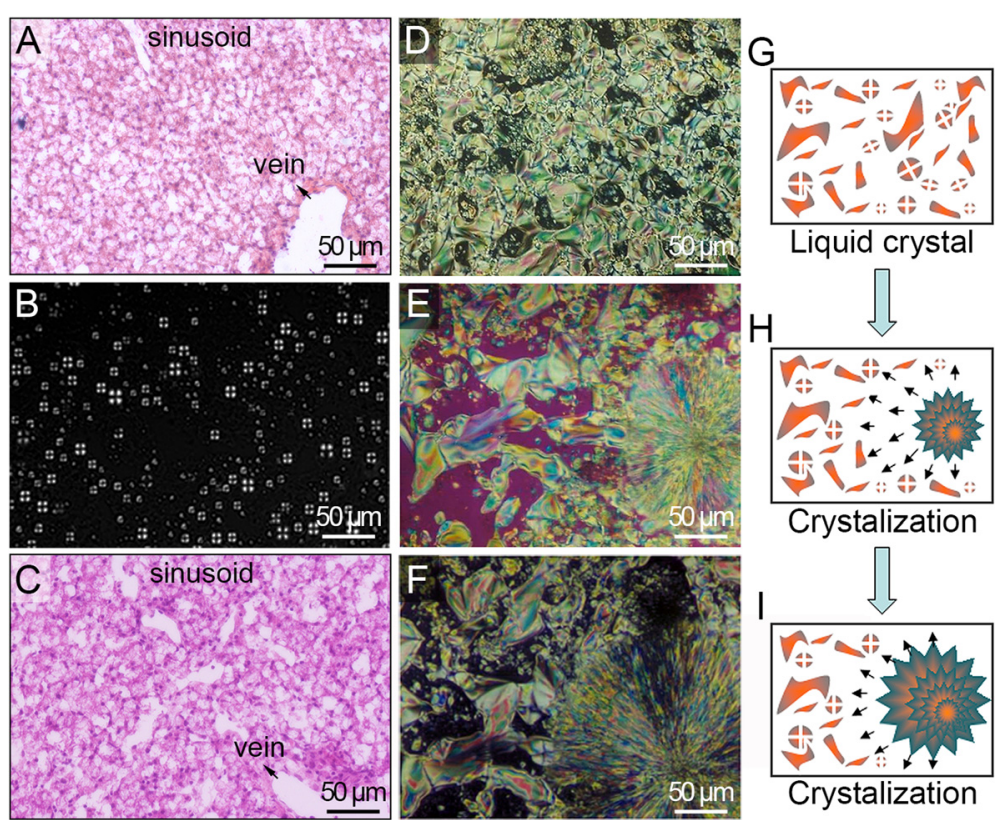

Fig. 4 Histological and phase-transitional characterization of enlarged livers from HiF diet Alb-NG37 mice. a Enlarged livers of HiF diet Alb-NG37 mice exhibited increased steatotic vesicles in hepatocytes in comparison to $\mathbf{c}$ the livers of normal-diet Alb-NG37mice. $\mathbf{b}$ The livers of HiF diet Alb-NG37 mice demonstrate Maltese cross activity. The transition from liquid crystal phase (e) to crystalline phase (d-f) was documented on samples extracted from enlarged Alb-NG37 livers. g-i A schematic of the crystallization process from an initiating "seed" crystal is presented in step to the photographed process in panels $\mathbf{d}-\mathbf{f}$. Scale bars are $50 \mu \mathrm{m}$ in length 
while hepatocytes of HiF diet nTG mice did not (not shown). In humans, steatotic vesicles are composed of triglycerides and known to exhibit liquid crystal properties. Thus, we performed phase transition and X-ray diffraction studies on the liquid crystals we isolated from HiF diet Alb-NG37 mouse livers to confirm the chemical composition of the hepatosteatosis.

As expected, temperature-catalyzed phase-transition studies conducted on Maltese cross birefringent active HiF diet Alb-NG37 livers maintained a liquid crystalline form and exhibited changes in birefringent texture typical of liquid crystals (Fig. 4d-f). Consistent with triglyceride behavior in previous developmental studies of cholesteryl, these liquid crystals also demonstrated the rapid transition from liquid crystal to full crystallization via nucleation from an initial "seed" crystal (schematic in Fig. 4g-i). These Maltese cross optical birefringent activities were morphologically similar to those found in hepatic liquid crystal lipid droplets (LCLDs) during chicken embryo development, indicating a reactivation of developmental pathways in fatty liver disease [13-19].

To confirm that the steatosis in our HiF diet Alb-NG37 is pathologically similar to human fatty liver disease, we determined the chemical composition of the steatotic vesicles via $\mathrm{X}$-ray diffraction (XRD) and small-angle $\mathrm{X}$-ray scattering (SAXS). The content of the steatotic vesicles in liquid crystal phase displayed a single $\mathrm{X}$-ray scattering peak at $2.44^{\circ}$ within the XRD $2 \theta$ detection spectrum of $0.6^{\circ}$ to $20^{\circ}$ and was confirmed with SAXS within detection spectrum of 0.3 to $20^{\circ}$ (Fig. 5a, b). By Bragg's equation, the $2.44^{\circ}$ value indicates a $36.8 \AA$ lattice plane distance $(\mathrm{d} \AA)$. The XRD diffraction analysis of the sample in its fully crystalline form identified diffractions at lattice planes $d(\AA)$ of $19.31,17.06,5.90,4.90,4.59$, and 4.09 corresponding to $I / I_{0}$ (100), $I / I_{0}$ (12), $I / I_{0}(10), I / I_{0}$ (35), $I / I_{0}$ (10), and $I / I_{0}$ (8) in the detection spectrum of $0.6^{\circ}$ to $45^{\circ}$ (Fig. $5 \mathrm{c}$ ). This XRD pattern matched the known diffraction of cholesteryl oleate (Fig. 5d and Table 1). Human fatty liver steatosis is also composed of cholesterol, indicating that hepatosteatosis in our HiF diet Alb-NG37 mice shares the pathology of human fatty liver disease.

\section{HiF diet Alb-NG37 TG mice exhibit cardiac arrhythmias}

Cardiac enlargement is a common symptom of many heart diseases. In order to better understand the physiological effects of doubled heart/body weight ratio in $\mathrm{HiF}$ diet Alb-NG37 transgenic mice, we performed electrocardiogram (ECG) on HiF diet Alb-NG37 mice. Prior to initiation of the HiF diet, Alb-NG37 mice had a cardiac rate of 375 beats per min (bpm) and RR interval of $160 \pm 35 \mathrm{~ms}$, which are comparable to the $400 \mathrm{bpm}$ and RR intervals of $150 \pm 10 \mathrm{~ms}$ seen in nTG littermate controls (Fig. 6a, b). However, Alb-NG37 mice rapidly developed severe bradycardia after initiation of the HiF diet (Fig. 6c). Not only is

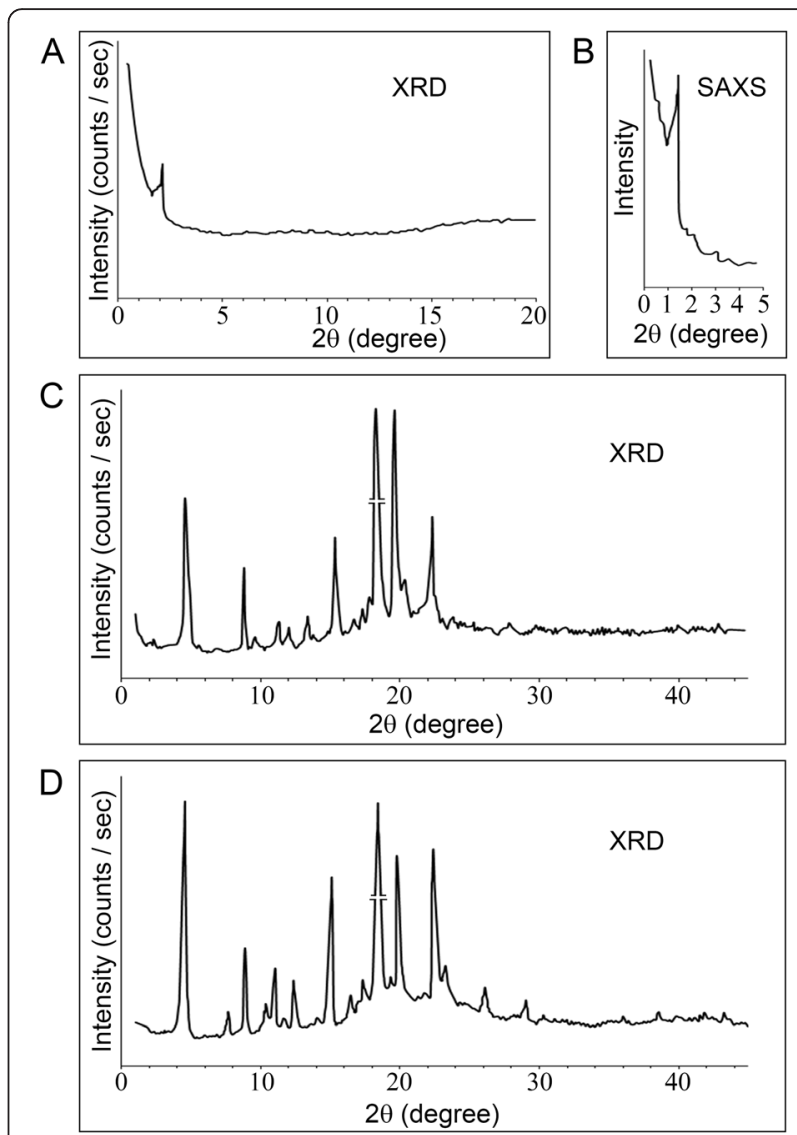

Fig. 5 XRD and SAXS characterization of extracts from steatotic vesicles of HiF diet Alb-NG37 mouse hepatocytes. Diffraction patterns of the extracts from HiF diet Alb-NG37 mice $(n=6)$ in liquid crystalline phase had a single diffraction peak by XRD diffraction angle (2 $\theta$ ) of $0.6-20^{\circ}$ (a) and SAXS angle (20) of $0.3-5^{\circ}(\mathbf{b})$. The XRD pattern of the same extracts in crystalline phase displayed a patterned set of diffraction peaks within XRD diffraction angle (20) of $0.6-45^{\circ}(n=4)(\mathbf{c})$, which is comparable to the standard XRD pattern of cholesteryl oleate (d)

the RR intervals in HiF diet Alb-NG37 mice extremely prolonged, but the RR intervals also followed a much wider distribution than both normal-diet Alb-NG37 mice and nTG littermates regardless of diet (Fig. 6d, e). HiF diet Alb-NG37 mice had mean RR intervals of $260 \pm 85 \mathrm{~ms}$ with $95 \%$ of RR intervals distributed between 200 and $330 \mathrm{~ms}$ with the full distribution of RR intervals ranging dramatically from 160 to $580 \mathrm{~ms}$ (Fig. 6d, e). This diet-dependent 3.6-fold difference in RR intervals within a single mouse is highly indicative of an irregular arrhythmia, which suggests a pathologic over physiologic bradycardia.

Arrhythmia is a common occurrence of aging [20, 21]. Bradycardia has been reported in younger patients and may not require immediate intervention if symptomatically mild [22, 23]. However, at 4 months of age, our transgenic mice are considered young adults. This young 
Table 1 X-ray diffraction pattern of the crystal transited from hepatic LCLDs in NG37 transgenic mouse

\begin{tabular}{lllll}
\hline \multicolumn{2}{l}{ Standard (cholesteryl oleate) } & & \multicolumn{2}{l}{ LCLC crystal, TG } \\
\cline { 1 - 2 }$/ / I_{0}$ & $d(\AA)$ & & $1 / I_{0}$ & $d(\AA)$ \\
\hline 100 & 19.34 & 100 & 19.31 \\
12 & 17.06 & 12 & 17.06 \\
4 & 10.54 & 4 & 10.60 \\
4 & 9.65 & 4 & 9.57 \\
3 & 8.32 & 3 & 8.28 \\
3 & 7.75 & 3 & 7.71 \\
3 & 6.91 & 3 & 6.91 \\
10 & 5.91 & 10 & 5.90 \\
2 & 5.28 & 2 & 5.23 \\
2 & 5.12 & 2 & 5.08 \\
35 & 4.91 & 35 & 4.90 \\
10 & 4.61 & 10 & 4.59 \\
8 & 4.11 & 8 & 4.09 \\
\hline
\end{tabular}

age in addition to the highly irregular nature of the bradycardia seen in our model likely generates symptomatic events (light-headedness, blurry vision, etc.) we cannot measure in mice. As such, the irregular bradycardia seen in our mice cannot be considered a normal physiological event but rather a pathological event triggered by $\mathrm{HiF}$ diet. Since this pathology was only found in HiF diet mice with overexpression of NG37 restricted to the liver, it is a reasonable conclusion that the fatty liver disease caused by the combination of HiF diet and NG37 overexpression is the underlying cause of this arrhythmia. Liver disease pathology may have indirectly led to the arrhythmia by causing the dramatic body habitus change that can lead to hypertension-induced cardiac enlargement. More likely, the severe cholesterol buildup in the steatotic livers of AlbNG37 is a symptom of HiF-diet-triggered imbalanced lipid metabolism, which can also cause abnormal accumulation of arterial plaques. These plaques can also lead to electrical conduction disturbances caused by small infarctions damaging the atrioventricular node, sinoatrial node, or Purkinje fibers. A partial block with some successful conduction compensated by a slower escape pacemaker would explain the highly irregular bradycardic rhythm. While we have not yet observed sudden cardiac death resulting from bradycardia-induced oxygen deprivation or from major myocardial infarctions, these mice may be more susceptible to such pathologies as they age.

\section{The diet-associated function of NG37 could be universal in mammals}

The human protein encoded by NG37 shares 86,83 , and $82 \%$ identity with its bovine (Bos Taurus), porcine (Sus
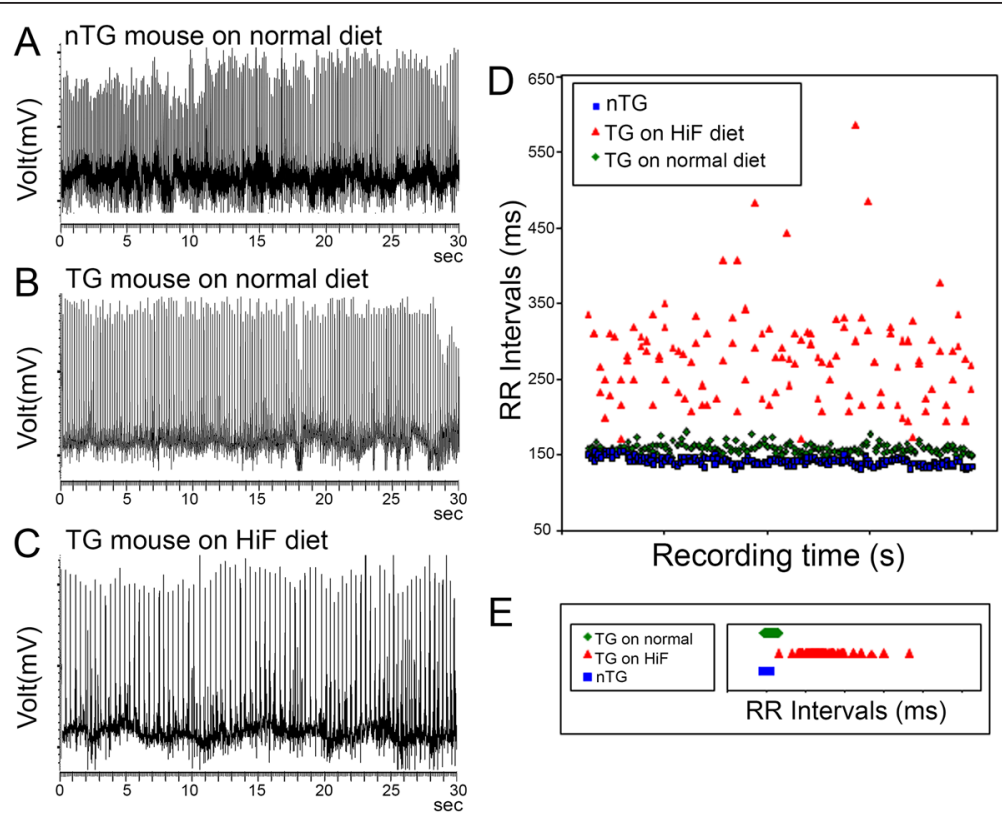

$\mathrm{E}$

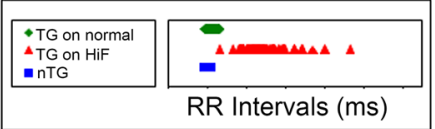

Fig. 6 ECG and RR intervals of HiF diet Alb-NG37 mice in comparison to normal-diet Alb-NG37 mice and normal-diet nTG littermates. a Normal-diet nTG littermates have EC tracings and heart rates comparable to $\mathbf{b}$ ECGs of normal-diet Alb-NG37 mice. c HiF diet Alb-NG37 mice have a significantly decreased heart rate noticeable on ECG tracing. d, e The RR interval of $160 \pm 35$ ms in normal-diet Alb-NG37 mice (green diamond) is consistent over time and comparable to the $150 \pm 10 \mathrm{~ms}$ in normal-diet nTG mice (blue square) whereas HiF diet Alb-NG37 TG mice (red triangle) have RR intervals of $260 \pm 85$ ms that range from 160 to 580 ms over time with $95 \%$ of RR intervals distributed between 200 and 330 ms 
Table 2 Schematic of the NG37 gene and its homology

\begin{tabular}{|c|c|c|c|c|c|}
\hline Species & Gene Name & NCBI & cDNA & Protein & Gene Conserved of Homology \\
\hline H. sapiens & $N G 37$ & NP_079534.2 & $2676 \mathrm{bp}$ & 891 aa & \\
\hline P. troglodytes & es $N G 37$ & XP_518359.3 & $2670 \mathrm{bp}$ & 889 aa & \\
\hline M. mulatta & G7c-like & XP_002808423.1 & $2712 \mathrm{bp}$ & 903 aa & \\
\hline C. lupus & NG37 & XP_849170.1 & $2670 \mathrm{bp}$ & 889 aa & \\
\hline B. taurus & $N G 37$ & NP_001068674.1 & $2676 b p$ & 891 aa & \\
\hline M. musculus & $N G 37$ & NP_613048.1 & $2676 \mathrm{bp}$ & 891 aa & \\
\hline R. norvegicus & s NG37 & NP_997664.1 & $2679 \mathrm{bp}$ & 892 aa & \\
\hline$X$. tropicalis & $N G 37$ & XP_004916972.1 & $2877 \mathrm{bp}$ & 958 aa & \\
\hline D. rerio & $\begin{array}{l}\text { si:ch73- } \\
235 d 11.1\end{array}$ & XP_003200526.2 & $3033 \mathrm{bp}$ & 1010 aa & \\
\hline
\end{tabular}

NOTES: VWA domain (von Willebrand factor A domain containing 7)

Het-C domain (Heterokaryon incompatibility protein)

scrofa), and murine (Mus musculus) equivalents, respectively. The highly conserved nature of the NG37 gene indicates that the function of this protein is crucial for mammalian life (Table 2). The function of the amino acid terminal vWA domain and carboxyl terminal MIDAS domain provides hints about the function of NG37; we still do not know the exact role of this gene. Although genotype/phenotype comparison and histocompatibility studies have associated the NG37 gene to lung tumor susceptibility, no causative associations have been proven $[4,24,25]$. Our findings in this liver-specific NG37 gain-of-function model elucidate a definitive function for NG37 in dietary cholesterol processing. The data revealed a HiF-diet-triggered pathology (fatty liver, cardiac enlargement, and cardiac arrhythmias) in mice overexpressing NG37 in the liver. This diet-associated phenotype argues that overexpression of NG37 disrupts cholesterol metabolism, resulting in hepatosteatosis, increased body habitus, and cardiac disease.

As a critical component of the cell membrane essential to maintaining the two-dimensional fluid membrane [26], cholesterol and its derivatives are often observed as massive birefringent Maltese crosses throughout the embryo and are found most abundantly in the liver $[15,17]$. After embryonic development, birefringent Maltese crosses are only seen in pathology associated with erroneous reactivation of embryonic pathways. Amongst these diseases are age-related macular degeneration [27], steatohepatitis and atherosclerosis [28], Anderson-Fabry disease $[14,15]$, and cytoplasmic accumulation in macrophage- monocytes $[14,15]$. The accumulation of liquid crystals in steatohepatitis was first observed in patients and later characterized in an animal model $[28,29]$. Our data show that overexpression of NG37 in the presence of a HiF diet can also lead to liquid crystal accumulation in the liver pathologically similar to steatohepatitis. The reappearance of liquid crystal-forming cholesterol derivatives in the livers of our HiF diet NG37 mice indicates the reactivation of normal embryonic developmental mechanisms in a pathological process similar to those seen in the other liquid crystal-related pathologies. As previously discussed, the steatotic vesicles in hepatocytes may also be a symptom of the same lipid metabolism imbalance that lead to arterial plaque accumulation and cardiac disease. The Maltese cross birefringent active steatotic droplets could be composed of LCLDs produced to buffer and protect the body from lipid and cholesterol accumulation. Thus, there are two alternative mechanisms behind this previously unknown association between NG37 and fatty liver the disease: (1) a primary pathogenic process caused by NG37 overexpression or (2) a pathogenic outcome from over activation of a protective mechanism.

\section{Conclusions}

HiF diet triggers the formation of massive cholesterolbased liquid crystals in the hepatocytes of our liver-specific NG37-overexpressing mouse model. This pathology led to fatty liver disease that is not seen in HiF diet nTG littermates, indicating that NG37 plays a role in cholesterol metabolism and homeostasis. When triggered, pathologic 
effects of NG37 overexpression were not restricted to the liver alone. HiF diet Alb-NG37 mice also suffered from an increased body habitus, a symptom expected in a mouse with disrupted lipid homeostasis. Interestingly, these mice also developed cardiac hypertrophy with heart/body weight ratios double than their HiF diet littermate controls. This could be explained by cardiac compensation for increased body habitus-induced hypertension, a leading cause of pathologic cardiac hypertrophy. However, the speed with which this phenotype developed needs to be further studied as it could be due to hypercholesterolemia-induced vessel disease or neurohormonal changes. In fact, coronary artery infarct due to plaque buildup is the most likely cause of the irregular bradycardia we also characterized in this model. The wide RR interval distribution in these mice indicates sinoatrial node dysfunction or conduction failure as the most probable causes. Since we confirmed that our transgene is only expressed in the liver, damage to electrical transduction in the heart is most likely due to micro infarctions caused by diet-induced hypercholesterolemia in Alb-NG37 mice. These findings indicate that overexpression of NG37 denotes latent risk for fatty liver disease and its subsequent sequelae that is only uncovered in the presence of a $\mathrm{HiF}$ diet. With the increasing quality of life, rich diets that were previously unavailable to our ancestors have led to increasing public health concerns over expanding waistlines and metabolic diseases. However, few direct links between a specific gene and dietary habits have been described. Here, we describe a gene, NG37, overexpression of which could predispose a patient to early onset nonalcoholic fatty liver disease, increased body habitus, and cardiac disease when triggered by a HiF diet.

\section{Methods}

\section{Animal maintenance and diet}

DBA/-2J and C57BL/6J mice (The Jackson Laboratory; Bar Harbor, ME) and the genetically modified mice generated in this study were bred under standard conditions for maintenance. For sample collection, animals were euthanized by cervical dislocation prior to dissection. Tissues for RNA extraction were harvested immediately and snap frozen with liquid nitrogen, while tissues for histological analysis were fixed with $4 \%$ paraformaldehyde in PBS immediately after collection. Samples for optical analysis were cryosectioned without delay. All animals were maintained in natural day-night cycle. All animal procedures were conducted under guidelines approved by the Animal Care and Use Committee of Xi'an for Animal Use of Universities in Shaanxi Province, P.R. China.

Experimental dietary alterations began when mice were 2 months of age. The control diet consisted of regular mouse chow (China National Standard GB14924.3-2010) purchased from Xi'an Fengwei Animals, Ltd. (Xi'an, China). HiF diet chow was produced in the following steps. First, a corn oil plus $5 \%$ cholesterol (CCh; Sigma-Aldrich) solution homogenized by heating corn oil to boiling temperature then adding cholesterol and maintaining boiling temperatures for $15 \mathrm{~min}$ for full cholesterol dissolution. Regular mouse chow was then fried in the HiF solution for about $3 \mathrm{~min}$ to generate the fat-enriched foods. The fried $\mathrm{HiF}$ foods were considered ready to serve after cooling to room temperature. The HiF diet chow replaced normal chow in HiF diet groups. Both diets were provided on a free-taking principle.

\section{Cloning, construction of NG37, and model animal generation for liver-specific overexpression of NG37}

Total RNA was extracted from mouse liver with Trizol (Life Technology) and evaluated by $0.7 \%$ agarose electrophoresis for 28S RNA, 18S RNA, and 5.8S RNA. Reverse transcription polymerase chain reaction (RT-PCR) was employed to amplify the cDNA sequence of NG37 from the total RNA. Poly(A)-RNA from total RNA was used to generate the first-strand cDNA with the cDNA synthesis kit according to the manufacturer's instructions (Gibco BRL, Gaithersburg, MD). Based on the availability of the convenient endonuclease restriction sites, primer pairs (forward primer TTGCGGCCCTCCCTGTGGAGGTACCC and reverse primer CTGTCGACGGCTGCGTTGAGGGCCTC) were designed according to the published cDNA sequence for mouse NG37 (ACCESSION: NM_138582 XM_994838). Not I and Sal I restriction sites were included in the forward primer and the backward primer at their $5^{\prime}$ ends, respectively, for ease of subcloning into pBlueScript II (+).

Due to lack of antibodies against NG37, a M2 FLAG (Asp-Tyr-Lys-Asp-Asp-Asp-Asp-Lys) was inserted before NG37 in the reading frame within the expression construct. Monoclonal antibody of M2-FLAG would be used to distinguish the overexpression of the protein. The gene was subcloned under the control of the albumin promotor for liver-specific expression. SV40 early region transcription terminator and polyadenylation site were placed at the 3'-UTR. The primer sequences used to generate M2FLAG::NG37 fusion cDNA were 5'TTGCGGCCGCCAC CATGGATTACAAGGATGACGACGATAAGCTCCCTG TGGAGGTACCCCTGTCCCACCTG3' and 5'CTGTCG ACGGCTGCGTTGAGGGCCTCGCCCATACTG CC3'. Again, Not I and Sal I restriction sites on the $5^{\prime}$ of M2FLAG and 3' of NG37 cDNA, respectively, were employed to insert the fusion cDNA into the Alb-SV40 polyA expression plasmid backbone. Highly purified Alb-NG37 minigene made by $\mathrm{CsCl}$ purification was then prepared for pronuclei injection. The minigene was microinjected into C57BL/6J zygotes, after which the injected zygotes were implanted into the oviducts of pseudopregnant DBA/-2J. Alb-NG37 transgenic mice were maintained in the DBA/-2J inbred strain. 


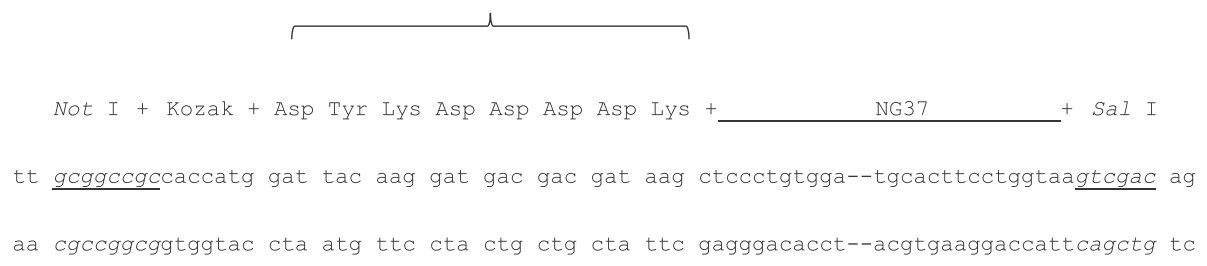

\section{RT-PCR, western blot, and northern blot}

Total RNA was extracted from the mouse liver with Trizol (Life Technology). First-strand cDNA was obtained with the cDNA synthesis kit as described above. Protein samples for western blot analysis extracted from mice were separated on a $15 \%$ Tris-glycine gel (Life Technology) and then transferred onto a polyvinylidene difluoride (PVDF) membrane. After blocking with $5 \%$ non-fat milk in TBST, membranes were detected with a primary rabbit anti-flag antibody (Sigma-Aldrich) followed by a secondary goat anti-rabbit IgG conjugated with horseradish peroxidase (HRP) and developed after treatment with Supersignal West Pico Chemiluminescent Substrate (Pierce Biotechnology) as previously described [17].

For northern blot analyses, RNA extracted from tissue were run in $4 \%$ formaldehyde agarose with formalin. Then, RNA were transferred onto nitrocellulose membrane and hybridized with H3-CTP-labeled M2-FLAG cDNA probe to visualize TG mRNA expression.

\section{Optical activity analyses and histology analyses}

Cryosections were used for optical activity analyses. After dissection of mouse liver, the samples were set into cryomatrix embedding reagent (OCT) and placed in an aluminum foil basket. Samples were frozen by dipping the foil basket into liquid nitrogen. The frozen OCT block was then cut at thickness of 10-30 m. The sections were mounted with $20 \%$ glycerol in PBS (pH 7.2) for polarization observation [14, 15, 17].

The samples obtained from the cryosection preparation were evaluated via two methods. First, conventional observation was carried out under non-crossed polarizer and analyzer to identify the distribution of birefringent activities. Then, birefringence in Maltese crosses of crystalline liquid crystal was documented. The observations were made on a XS-213A-P Polarizing Microscope (Jiangnan Jnoec Ltd., Nanjing).

Liquid crystal extracts were carried out according to previously published protocols [14, 15, 17]. Histological analysis was carried out on tissues fixed with $4 \%$ neutralbuffered paraformaldehyde. The samples were processed for paraffin section and stained with hematoxylin and eosin $(H \& E)$ as previously published $[13,16]$.

\section{SAXS and XRD}

For characterization of liquid crystalline samples, the content of steatotic vesicles were extracted from liver samples of HiF diet Alb-NG37 mice and examined as previously described (Xu MM et al. 2012). SAXS patterns of the samples were obtained on the small-angle goniometer of $\mathrm{D} / \mathrm{max}$-rA diffractometer in diffraction/scattering angle $(2 \theta)$ of $0.6-20^{\circ}$ and $0.3-5^{\circ}$ with $\mathrm{CuK} \alpha$ radiation, Ni filter, $50 \mathrm{kV} \times 100-120 \mathrm{~mA}$, and slit sizes 0.16-0.12-0.2-0.4 mm. Samples were clutched between two non-diffraction films to reduce movement and dehydration.

To further confirm the chemical composition as cholesterol derivatives, content of steatotic vesicles were extracted from liver samples of HiF diet Alb-NG37 mice then crystallized following a previously described protocol $[15,17]$. The XRD patterns of the crystal samples were conducted on the wide-angle goniometer of $\mathrm{D} /$ max-rA diffractometer in diffraction angle $(2 \theta)$ of $0.6-45^{\circ}$ with $\mathrm{CuK \alpha}$ radiation, graphite monochromator, and slit sizes of $1 / 6^{\circ}-1 / 6^{\circ}-0.15-0.45 \mathrm{~mm}$. The results were then compared to the XRD pattern of cholesteryl oleate.

\section{Electrocardiographical analyses}

Mice undergoing telemetric electrocardiographical measurements (Data Sciences International, St. Paul, MN) were anesthetized with $1.5 \%$ isoflurane, and electrodes were surgically positioned in the intraperitoneal cavity following the lead II configuration according to manufacturer's description [13]. Data were collected using an implantable radiofrequency micro-transmitter (TA1010ETAF20) and recorded continuously using the waveform format hardware modification for multiple instrumented mice. The data analyses were performed using Dataquest A.R.T. version 4.1 (Data Sciences International).

\section{Ethics approval and consent to participate}

All applicable international, national, and/or institutional guidelines for the care and use of animals were followed.

\section{Availability of data and material}

The data set supporting the conclusions of this article are included within the article. 


\section{Abbreviations}

bpm: beats per min; ECG: electrocardiogram; F6/H1: fibulin-6/hemicentin-1; F8/H2: fibulin-8/hemicentin-2; H\&E: hematoxylin and eosin; HiF: high-fat; HRP: horseradish peroxidase; LCLDs: liquid crystal lipid droplets; nTG: nontransgenic; OCT: cryomatrix embedding reagent; PVDF: polyvinylidene difluoride; RT-PCR: reverse transcription polymerase chain reaction; TG: transgenic; WA: von Willebrand A.

\section{Competing interests}

The authors declare that they have no competing interests.

\section{Authors' contributions}

XXH conceived of the study. ZX, MMX, WLY, MYL, FR, DZL, PYX, CX, and LYF developed protocols and collected and analyzed all data. XXH, ZX, MMX, WLY, ZS, DA, JM, and WI prepared the manuscript, and all authors edited the manuscript. All authors read and approved the final manuscript.

\section{Acknowledgements}

This work was supported by the National Natural Science Foundation of China (Grant No. 31371256, 31571273), the Ministry of Science and Technology of China (Grant No.2015CB943100), the National Department of Education Central Universities Research Fund (Grant No. GK20130100), the Foreign Distinguished Scientist Program (Grant No. MS2014SXSF038), US Maryland Stem Cell Research Fund (2009MSCRFE008300), Qinba Mountain Developing Center (Grant No. CIC-QBRSD), and the Outstanding Doctoral Thesis fund (Grant No. X2014YB02, X2015YB05).

\section{Author details}

${ }^{1}$ College of Life Sciences, Shaanxi Normal University, Xi'an, Shaanxi 710062, China. ${ }^{2}$ Department of Pharmacology, Duke University Medical Center, Durham, NC 27708, USA. ${ }^{3}$ State Key Laboratory for Animal Nutrition, Institute of Animal Science, Chinese Academy of Agricultural Sciences, Beijing 100193, China. ${ }^{4}$ Lanzhou University School of Medicine, Lanzhou 730030, China. ${ }^{5}$ Case Western Reserve University School of Medicine, Cleveland, $\mathrm{OH} 44106$, USA. ${ }^{6}$ Wuhan General Hospital of Guangzhou Military Command, Wuhan, Hubei 430070, China. ${ }^{7}$ College of Food Engineering and Nutritional Science, Shaanxi Normal University, Xi'an, Shaanxi 710062, China. ${ }^{8}$ Ohio State University School of Medicine, Columbus, OH 43210, USA. ${ }^{9}$ Johns Hopkins School of Medicine, Baltimore, MD 21287, USA

\section{Received: 29 November 2015 Accepted: 25 April 2016}

\section{Published online: 21 May 2016}

\section{References}

1. Anderson N, Borlak J. Molecular mechanisms and therapeutic targets in steatosis and steatohepatitis. Pharmacol Rev. 2008;60:311-57. doi:10.1124/pr.108.00001.

2. Billings LK, Florez JC. The genetics of type 2 diabetes: what have we learned from GWAS? Ann N Y Acad Sci. 2010;1212:59-77. doi:10.1111/j.1749-6632. 2010.05838.x

3. Hutter H, Vogel BE, Plenefisch JD, Norris CR, Proenca RB, Spieth J, et al. Conservation and novelty in the evolution of cell adhesion and extracellular matrix genes. Science. 2000;287:989-94. doi:10.1126/science.287.5455.989.

4. Kumánovics A, Lindahl KF. G7C in the lung tumor susceptibility (Lts) region of the Mhc class III region encodes a von Willebrand factor type A domain protein. Immunogenetics. 2001;53:64-8. doi:10.1007/s002510000297.

5. Perricone C, Ciccacci C, Ceccarelli F, Di Fusco D, Spinelli FR, Cipriano E, et al. TRAF3IP2 gene and systemic lupus erythematosus: association with disease susceptibility and pericarditis development. Immunogenetics. 2013;65:703-9. doi:10.1007/s00251-013-0717-6.

6. Whittaker CA, Hynes RO. Distribution and evolution of von Willebrand/ integrin A domains: widely dispersed domains with roles in cell adhesion and elsewhere. Mol Biol Cell. 2002;13:3369-87. doi:10.1091/mbc.E02-05-0259.

7. C. elegans Sequencing Consortium. Genome sequence of the nematode $C$. elegans: a platform for investigating biology. Science. 1998;282:2012-8. doi:10.1126/science.282.5396.2012.

8. Vogel BE, Hedgecock EM. Hemicentin, a conserved extracellular member of the immunoglobulin superfamily, organizes epithelial and other cell attachments into oriented line-shaped junctions. Development. 2001;128:883-94.

9. Feitosa NM, Zhang J, Carney TJ, Metzger M, Korzh V, Bloch W, et al. Hemicentin 2 and fibulin 1 are required for epidermal-dermal junction formation and fin mesenchymal cell migration during zebrafish development. Dev Biol. 2012;369:235-48. doi:10.1016/j.ydbio.2012.06.023.

10. Jordan SN, Olson S, Canman JC. Cytokinesis: thinking outside the cell. Curr Biol. 2011;21:R119-21. doi:10.1016/j.cub.2010.12.040.

11. Xu X, Vogel BE. A secreted protein promotes cleavage furrow maturation during cytokinesis. Curr Biol. 2011;21:114-9. doi:10.1016/j.cub.2010.12.006.

12. Xu X, Xu M, Zhou X, Jones OB, Moharomd E, Pan Y, et al. Specific structure and unique function define the hemicentin. Cell Biosci. 2013;3:27-32. doi:10. 1186/2045-3701-3-27.

13. Maruyama M, Li BY, Chen H, Xu X, Song LS, Guatimosim S, et al. FKBP12 is a critical regulator of the heart rhythm and the cardiac voltage-gated sodium current in mice. Circ Res. 2011;108:1042-52. doi:10.1161/CIRCRESAHA.110.237867.

14. Xu M, Jones OD, Zheng S, Li Y, Yan G, Pan Y, et al. Cytoplasmic accumulation of liquid-crystal like droplets in post-infection sputum generated by gram-positive bacteria. Mol Cryst Liq Cryst. 2011;547:173-80. doi:10.1080/15421406.2011.572045.

15. Xu M, Xu X. Liquid-crystal in embryogenesis and pathogenesis of human diseases. In: Sato K, editor. Embryogenesis. Rijeka: InTech; 2012. p. 637-52.

16. Xu X, Dong C, Vogel BE. Hemicentins assemble on diverse epithelia in the mouse. J Histochem Cytochem. 2007;55:119-26. doi:10.1369/jhc.6A6975.2006.

17. Xu X, Xu M, Jones OD, Chen X, Li Y, Yan G, et al. Liquid crystal in lung development and chicken embryogenesis. Mol Cryst Liq Cryst. 2011;547: 164-72. doi:10.1080/15421406.2011.572042.

18. Bharati S, Goldschlager N, Kusumoto F, Lazzara R, Azar R, Hammill S, et al. Sinus node dysfunction. In: Camm AJ, Saksena S, editors. Electrophysiological disorders of the heart. Phildadelphia: Elsevier Churchill-Livingstone; 2005. p. 207-26. doi:10.1016/B978-0-443-06570-5.50018-6.

19. Vogler J, Breithardt G, Eckardt L. Bradyarrhythmias and conduction blocks. Rev Esp Cardiol (Engl Ed). 2012;65:656-67. doi:10.1016/j.rec.2012.01.027.

20. Kumar P, Kusumoto FM, Goldschlager N. Bradyarrhythmias in the elderly. Clin Geriatr Med. 2012;28:703-15. doi:10.1016/j.cger.2012.08.004.

21. Santos MA, Sousa AC, Reis FP, Santos TR, Lima SO, Barreto-Filho JA. Does the aging process significantly modify the mean heart rate? Arq Bras Cardiol. 2013;101:388-98. doi:10.5935/abc.20130188.

22. Mason KP, Lonnqvist PA. Bradycardia in perspective-not all reductions in heart rate need immediate intervention. Paediatr Anaesth. 2015;25:44-51. doi:10.1111/pan.12584

23. Oflaz MB, Kucukdurmaz Z, Guven AS, Karapinar H, Kaya A, Sancakdar E, et al. Bradycardia seen in children with Crimean-Congo hemorrhagic fever. Vector Borne Zoonotic Dis. 2013;13:807-11. doi:10.1089/vbz.2012.1200.

24. Snoek M, Albertella MR, van Kooij M, Wixon J, van Vugt $H$, de Groot $K$, et al. G7c, a novel gene in the mouse and human major histocompatibility complex class III region, possibly controlling lung tumor susceptibility. Immunogenetics. 2000;51:383-6. doi:10.1007/s002510050634.

25. van Kooij M, de Groot K, van Vugt H, Aten J, Snoek M. Genotype versus phenotype: conflicting results in mapping a lung tumor susceptibility locus to the $\mathrm{G} 7 \mathrm{C}$ recombination interval in the mouse $\mathrm{MHC}$ class III region. Immunogenetics. 2001:53:656-61, doi:10.1007/s00251-001-0381-0.

26. Alberts B, Johnson A, Lewis J, Raff M, Roberts K, Walter P. Molecular biology of the cell. New York: Garland Science Press; 2008.

27. Haimovici R, Gantz DL, Rumelt S, Freddo TF, Small DM. The lipid composition of drusen, Bruch's membrane, and sclera by hot stage polarizing light microscopy. IOVS. 2001;42:1592-9.

28. Goldstein JL, Brown MS. From fatty streak to fatty liver: 33 years of joint publications in the JCI. J Clin Invest. 2008;118:1220-2. doi:10.1172/jci34973.

29. Goldstein $J$, Brown MS. The clinical investigator: bewitched, bothered, and bewildered-but still beloved. J Clin Invest. 1997:99:2803-12. doi:10.1172/jci119470. 